\title{
The levels of adipokines in relation to hormonal changes during the menstrual cycle in young, normal-weight women
}

Katarzyna Wyskida', Grzegorz Franik², Tomasz Wikarek³, Aleksander Owczarek4, Alham Delroba ${ }^{1}$, Jerzy Chudek $^{5}$, Jerzy Sikora ${ }^{6}$ and Magdalena Olszanecka-Glinianowicz ${ }^{1}$

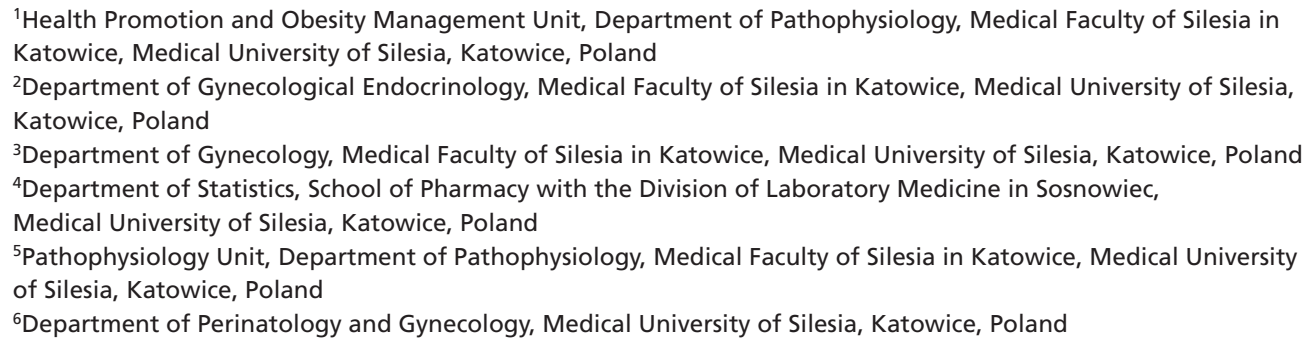

Correspondence should be addressed to M Olszanecka-

Glinianowicz

Email

magols@esculap.pl

\begin{abstract}
Context: The aim of this study was to assess the plasma leptin, adiponectin, resistin, visfatin/NAMPT, omentin-1, vaspin, apelin, TNF- $\alpha$, IL- 6 and RBP4 levels in relation to hormonal changes during the menstrual cycle in young, healthy, normal-weight women. Methods: The study involved 52 young, healthy, normal-weight women. Anthropometric parameters, body composition and levels of plasma leptin, adiponectin, resistin, visfatin/NAMPT, omentin-1, vaspin, apelin, TNF- $\alpha$, IL- 6 and RBP4 in addition to serum $\mathrm{FSH}, \mathrm{LH}$, estradiol, progesterone, 17-OH progesterone, androgens, SHBG and insulin concentrations were measured during a morning in fasting state three times: between days 2-4, days 12-14 and days 24-26 of the menstrual cycle.

Results: Plasma adiponectin, omentin-1, resistin and visfatin/NAMPT, apelin, TNF- $\alpha$, IL- 6 and RBP4 concentrations were stable during the menstrual cycle, while leptin and vaspin levels were significantly higher in both the midcycle and the luteal phases than those in the follicular phase. Multivariate regression analyses revealed that changes in leptin and vaspin levels between the follicular and the luteal phase are strongly related to changes in total testosterone levels.

Conclusions: Our results revealed stable levels of adipokines during the phases of the physiological menstrual cycle, except for leptin and vaspin, which showed increased levels in both the midcycle and the luteal phases. This effect was significantly associated with changes in the secretion of testosterone, $17-\mathrm{OH}$ progesterone and insulin in the luteal phase.
\end{abstract}

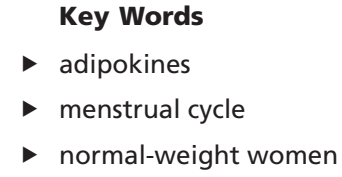

Endocrine Connections (2017) 6, 892-900

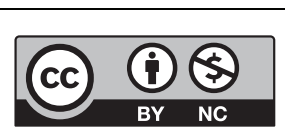

This work is licensed under a Creative Commons Attribution-NonCommercial 4.0 International License. 


\section{Introduction}

It has been shown that the levels of different adipokines, such as leptin, adiponectin, resistin, vaspin, omentin-1 and RBP4 are higher in women than those in men $(1,2,3,4,5,6)$, and that estrogens stimulate secretion of some adipokines, while androgens exert an opposite effect $(1,2,6)$. Additionally, it has been shown that some adipokines modulate reproductive functions, both indirectly by affecting the pituitary-ovary axis and directly by acting on ovarian receptors. The stimulation of leptin receptors in hypothalamus is necessary to activate the hypothalamus-pituitary-gonadal (HPG) axis to trigger puberty and maintain reproductive function (7). Also, the activation of leptin and adiponectin receptors in the pituitary gland have promoting effect on gonadotropin secretion, while the stimulation of apelin receptors have an opposite effect $(6,8)$. Furthermore, higher leptin levels have deleterious effect on ovarian steroidogenesis (7). These data suggest the existence of both positive and negative feedback between adipokines, gonadotrophins and ovarian hormones.

The results of previously published studies which assessed changes of selected adipokine levels during the physiological menstrual cycle were contradictory. For example, the higher circulating leptin level was found during the luteal phase by some researchers $(9,10,11$, 12); however, Cappobianco and coworkers (12) did not observe any changes in leptin levels during the menstrual cycle in both ovulatory and anovulatory women. Additionally, in some (13), but not in other studies (14), the association between changes in plasma leptin levels and variations of both estradiol and progesterone release were observed during the menstrual cycle. Similarly, the results of studies which assessed changes in resistin and adiponectin levels during the physiological menstrual cycle are ambiguous. Asimakopoulos and coworkers (10) observed that plasma adiponectin concentration remains stable during the menstrual cycle, while circulating resistin levels were higher in the luteal than in the follicular and the midcycle phase. However, Dafopoulos and coworkers (15) did not find any significant changes in circulating resistin levels during the physiological menstrual cycle. Furthermore, Galván and coworkers (16) have shown lower plasma adiponectin levels in the luteal than in the follicular and the midcycle phase. Moreover, the results of some studies revealed the lack of relationships between adiponectin or resistin levels and estradiol or progesterone concentrations in the circulation $(16,17,18,19)$. It seems that the inconsistencies in the previously cited data are the result of having small size of study cohorts, and in some cases, the inclusion of overweight women. The lack of TNF-alpha changes during the menstrual cycle has been observed in a single study (20), while plasma concentrations of omentin, visfatin/NAMPT, vaspin, apelin-36, RBP4 and IL-6 have not yet been studied during the physiological menstrual cycle. Finally, there were limited data available on the association between serum levels of ovarian steroids or gonadotrophins and plasma visfatin/NAMPT, vaspin, omentin-1, apelin-36, RBP4 and IL-6 in normal-weight women. Due to the existing inconsistencies in previously published studies, our study focused on the assessment of plasma leptin, adiponectin, resistin, visfatin/NAMPT, omentin-1, vaspin, apelin, TNF- $\alpha$, IL-6 and RBP4 levels in relation to hormonal changes during the menstrual cycle in young, healthy, normal-weight women.

\section{Material and methods}

The study involved 52 healthy, normal-weight women, aged 18-30 years, with regular menstrual cycles and a stable body mass index (BMI) during the 3-month period before the study. Patients diagnosed with polycystic ovary syndrome (PCOS), Cushing's syndrome, thyroid dysfunctions, androgen-secreting tumor, and enzyme deficiency (21-hydroxylase in particular), decreased ovary reserves, type 1 and 2 diabetes were excluded in our study. Any cases with pharmacological therapy, smoking or alcohol abuse were also excluded.

The study was conducted among patients referred from contraceptive consulting after obtaining the informed consent from each participant. The study protocol was approved by the Bioethical Committee of Medical University of Silesia in Katowice. Normal weight was defined according to WHO criteria (21) as BMI between 18.5 and $24.9 \mathrm{~kg} / \mathrm{m}^{2}$. The data concerning general and gynecological health were obtained on the basis of medical history as well as general and gynecological examinations. The characteristics of the study group are presented in Table 1.

Anthropometric measurements (body mass, height and waist circumference) were performed and BMI was calculated according to the standard formula. Body composition was assessed by the bioimpedance method, using Bodystat 1500 (Douglas, Isle of Man). After 16h of fasting overnight, $15 \mathrm{~mL}$ samples of venous blood were withdrawn in the morning between 8:00 and 9:00h. The blood samples were collected between days 2 and 
Table 1 Patients characteristics.

\begin{tabular}{|c|c|}
\hline & $N=52$ \\
\hline Age (years) & $21.5 \pm 1.5$ \\
\hline Body mass $(\mathrm{kg})$ & $58.5 \pm 5.4$ \\
\hline BMI $\left(\mathrm{kg} / \mathrm{m}^{2}\right)$ & $21.3 \pm 1.6$ \\
\hline Body fat (kg) & $15.9 \pm 2.9$ \\
\hline Body fat (\%) & $27.1 \pm 3.2$ \\
\hline Waist circumference $(\mathrm{cm})$ & $70.8 \pm 3.7$ \\
\hline Menarche (year of age) & $12.7 \pm 0.9$ \\
\hline Menstrual cycle duration (days) & $28.6 \pm 2.1$ \\
\hline
\end{tabular}

4 of the follicular phase, between days 12 and 14 of the midcycle phase and between days 24 and 26 of the luteal phase of each subject's menstrual cycle. These blood samples were collected according to recommendations of the kit's manufacturers. The serum and plasma samples were frozen and stored at $-70^{\circ} \mathrm{C}$.

\section{Laboratory procedures}

Serum follicle-stimulating hormone (FSH), luteinizing hormone $(\mathrm{LH})$, prolactin $(\mathrm{PRL})$, estradiol $\left(\mathrm{E}_{2}\right)$, total testosterone, free testosterone, androstendione, DHEA-S and SHBG were determined by ELISA (DRG Instruments $\mathrm{GmbH}$, Marburg, Germany). The respective intra- and inter-assay coefficients of variations were 5.5\% and $6.1 \%$ for FSH, 5.6\% and $6.2 \%$ for $\mathrm{LH}, 4.5 \%$ and $5.9 \%$ for PRL, $4.7 \%$ and $7.8 \%$ for $\mathrm{E} 2,3.6 \%$ and $7.1 \%$ for testosterone, $6.4 \%$ and $8.0 \%$ for free testosterone, $6.5 \%$ and $10.2 \%$ for androstendione, $4.8 \%$ and $7.5 \%$ for DHEA-S and $5.3 \%$ and 9.0\% for SHBG. Also, plasma glucose was estimated by colorimetric method using a commercially available test kit (Roche). Moreover, serum insulin and progesterone levels were assessed by the electrochemiluminescence method on the Cobas E411 analyzer (Roche Diagnostics $\mathrm{GmbH}$ ), with inter-assay coefficients of variability below $3.8 \%$ and below $11.8 \%$, respectively. In addition, HOMA-IR was calculated according to the standard formula: HOMA-IR=fasting serum insulin $(\mu \mathrm{IU} / \mathrm{mL}) \times$ fasting glucose $(\mathrm{mg} / \mathrm{dL}) / 405$. The cutoff value for insulin resistance was $\geq 2.5$.

The ELISA method was also used for measurements of plasma leptin (TECOmedical AG, Sissach, Switzerland), adiponectin (TECOmedical AG), resistin (R\&D Systems), visfatin/NAMPT (BioVendor, Brno, Czech Republic), omentin-1 (BioVendor), vaspin (BioVendor), apelin-36 (Phoenix Pharmaceuticals, Burlingame, CA, USA), TNF-alpha (R\&D Systems), IL-6 (R\&D Systems) and RBP4 (Phoenix Pharmaceuticals), with the lower limit of sensitivity of $0.2,0.6,0.05,0.03,0.5,0.01,0.11,0.18$,
0.02 and $2.17 \mathrm{ng} / \mathrm{mL}$, respectively. The intra- and interassay coefficients of variations were $7 \%$ and $8 \%$ for leptin, $5 \%$ and $6 \%$ for adiponectin, 5\% and 5\% for resistin, 9\% and $6 \%$ for visfatin/NAMPT, $4 \%$ and $5 \%$ for omentin- 1 and $9 \%$ and $10 \%$ for vaspin, $5.0-10.0 \%$ and $<15.0 \%$ for apelin-36, $14.4 \%$ and $18.7 \%$ for TNF-alpha, $7.4 \%$ and $7.8 \%$ for IL- 6 and $5.0 \%$ and $<14.0 \%$ for RBP 4 .

\section{Statistical analysis}

The statistical analyses were performed using STATISTICA 10.0 PL (StatSoft, Krakow, Poland) software and R software environment. The results are presented as mean values \pm s.D., and the distribution of variables was evaluated by the D'Agostino-Pearson test. The homogeneity of variances was assessed by the Levene test. Of note, the quantitative variables were compared with multivariate analysis of variances for repeated measurements with Tukey's post hoc test. In case of violence of the sphericity test, multivariate tests were applied. The assessment of associations between variables was performed with multivariate linear regression and the outliers were identified based on Cook's distance values. The Cook-Weisberg test was used to test the residuals for heteroskedasticity, and $\eta^{2}$ was used as the effect size estimate for the regression analysis. All the results were considered as statistically significant with a $P$ value of $<0.05$.

\section{Results}

As was expected, the serum FSH level was significantly higher in the midcycle than that in the follicular and the luteal phases, and it was higher in the follicular phase than that in the luteal phase of the menstrual cycle. Moreover, serum LH and estradiol levels were significantly increased in the midcycle than those in both the follicular and the luteal phases, and they were determined to be higher in the luteal than in the follicular phase. Serum progesterone and total testosterone levels were significantly lower in the follicular phase than in both the midcycle and the luteal phases of the menstrual cycle. Additionally, serum SHBG levels were significantly lower in the midcycle than in both the follicular and the luteal phases of the menstrual cycle (Table 2). However, there was no difference in insulin levels during the menstrual cycle phases (Table 2). Plasma adiponectin, omentin-1, resistin, visfatin/NAMPT, apelin-36, TNF- $\alpha$, IL- 6 and RBP4 concentrations were stable, while leptin and vaspin levels http://www.endocrineconnections.org DOI: 10.1530/EC-17-0186
() 2017 The authors Published by Bioscientifica Ltd
This work is licensed under a Creative Commons Attribution-NonCommercial 4.0 International License. 

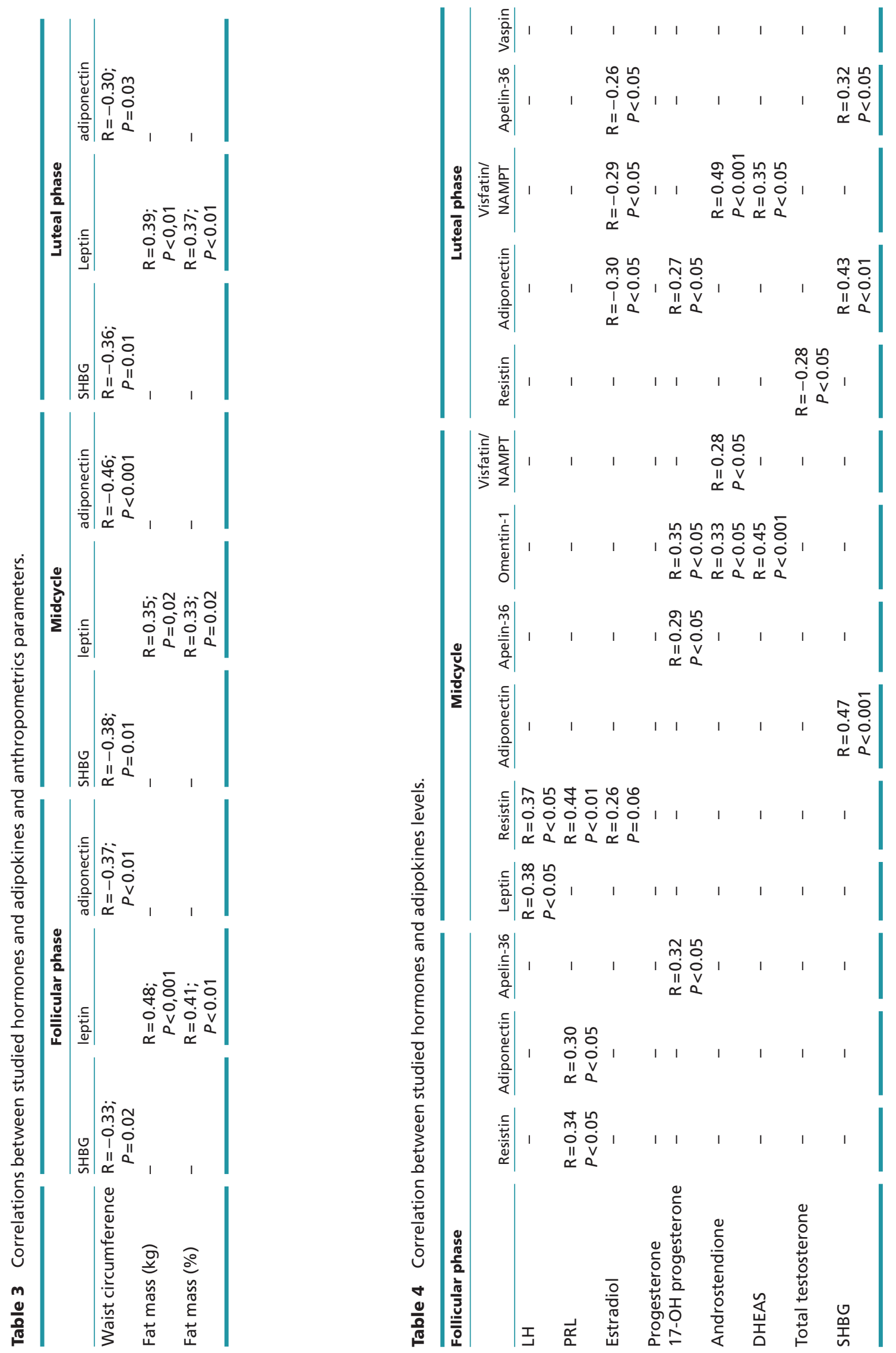
Table 5 Results of multivariable stepwise backward linear regression in assessment between differences in changes of leptin or vaspin and $\mathrm{FSH}, \mathrm{LH}$, estradiol, progesterone, 17- $\mathrm{OH}$ progesterone, total testosterone and insulin levels.

\begin{tabular}{l}
$\Delta \mathbf{2}$ \\
\hline Variables \\
\hline Total testosterone $(\mathrm{ng} / \mathrm{mL})$ \\
$17-\mathrm{OH}$ progesterone $(\mathrm{ng} / \mathrm{mL})$ \\
Insulin $(\mu \mathrm{lU} / \mathrm{mL})$ \\
Constants
\end{tabular}

\begin{tabular}{|c|c|c|c|}
\hline \multicolumn{4}{|c|}{ Leptin } \\
\hline$\beta \pm 95 \% \mathrm{Cl}$ & $t$ & $P$ & $\eta^{2}$ \\
\hline $6.22(1.72-10.72)$ & 2.77 & $<0.01$ & 29.1 \\
\hline $1.88(0.12-3.64)$ & 2.14 & $<0.05$ & 26.3 \\
\hline $0.64(0.15-1.13)$ & 2.63 & $<0.05$ & 17.4 \\
\hline $2.11(-1.14-5.36)$ & 1.30 & 0.20 & - \\
\hline
\end{tabular}

\begin{tabular}{|c|c|c|c|}
\hline \multicolumn{4}{|c|}{ Vaspin } \\
\hline$\beta \pm 95 \% \mathrm{Cl}$ & $t$ & $P$ & $\eta^{2}$ \\
\hline $0.136(0.061-0.213)$ & 3.60 & $<0.01$ & 41.5 \\
\hline $0.031(0.001-0.061$ & 2.08 & $<0.05$ & 13.9 \\
\hline $0.009(0.001-0.175)$ & 2.25 & $<0.05$ & 16.2 \\
\hline $0.009(-0.046-0.064)$ & 0.32 & 0.75 & - \\
\hline
\end{tabular}

serum (Table 4). There were positive correlations between serum SHBG and plasma adiponectin levels in both the midcycle and the luteal phases. Moreover, in the luteal phase, only a positive correlation was found between serum SHBG concentrations and plasma vaspin levels (Table 4). There was a correlation between HOMA-IR values and resistin levels in the follicular phase $(\mathrm{R}=0.35)$, between HOMA-IR values and omentin- $1(\mathrm{R}=0.28)$, and visfatin/NAMPT $(\mathrm{R}=-0.28)$ and $\mathrm{RBP} 4$ levels $(\mathrm{R}=0.33)$ in the midcycle, as well as a correlation between HOMA-IR values and leptin levels $(\mathrm{R}=0.41)$ in the luteal phase.

\section{Multivariable regression for changes of leptin and vaspin}

For further analysis of data, two additional classes of variables were created to represent changes in variables between the midcycle and the follicular phase $\left(\Delta_{1}\right)$ and the changes between the luteal phase and the follicular phase $\left(\Delta_{2}\right)$. A stepwise backward multivariable linear regression was performed to assess the relationships between changes of plasma leptin or vaspin levels and serum FSH, LH, estradiol, progesterone, $17-\mathrm{OH}$ progesterone, total testosterone and insulin levels.

The results of our analysis concerning $\Delta_{1}$ changes indicated no statistically significant correlation between changes of leptin and vaspin and the hormones mentioned above, while for $\Delta_{2}$ changes, significant correlations were found between changes of leptin and vaspin and changes in total testosterone, 17-OH progesterone and insulin levels (Table 5). Based on the $\eta^{2}$ values, the most notable relationship was between the changes in plasma leptin and vaspin levels and the changes in total testosterone levels between the luteal and follicular phases.

\section{Discussion}

To the best of our knowledge, our study is the first attempt to assess the changes of omentin-1, vaspin, visfatin/ NAMPT, apelin-36, RBP4 and IL-6 during the physiological menstrual cycle in young, healthy, normal-weight women. The results of our study revealed that plasma adiponectin, resistin, omentin-1, visfatin/NAMPT, apelin-36, RBP4, IL-6 and TNF- $\alpha$ levels were stable during the menstrual cycle, while plasma vaspin levels were increased in both the midcycle and the luteal phases compared to the follicular phase; these increases were proportional to the changes in estrogen and 17-OH progesterone levels. Although we did not observe any associations between plasma vaspin level and either gonadotrophins or sex steroid hormones, the results of multivariate analysis revealed that changes of vaspin levels between the follicular and the luteal phase were associated with changes in total testosterone and $17-\mathrm{OH}$ progesterone levels as well as HOMA-IR values. This hypothesis is supported by the results of previously published studies, which revealed a reduction in adipocyte insulin receptor binding (22) and a decrease in insulin sensitivity in the luteal phase in comparison to the follicular phase of the menstrual cycle (23). Additionally, the hypothetical insulin-sensitizing effect of vaspin would be in line with positive correlation between levels of vaspin and SHBG in the luteal phase, observed in this study.

Additionally, in accordance with the most previously published studies $(9,10,11)$, we observed increased leptin levels in both the midcycle and the luteal phases. It should be emphasized that midcycle serum LH concentrations were proportional to the circulating leptin levels, corroborating the role of leptin in the regulation of reproductive function (7). Moreover, plasma leptin levels were not associated with either estradiol or progesterone concentrations, which is in line with the results of some (13), but not other studies (14). However, the results of multivariate analysis revealed that changes in leptin levels between follicular and luteal phase are associated with the changes of total testosterone and $17-\mathrm{OH}$ progesterone levels as well as HOMA-IR values. These results suggest that vaspin and leptin may play a role not only in hormonal changes during the menstrual cycle but also indirectly in the regulation of sexual activity in women. This hypothesis is partially supported by a study performed by Caruso and 
coworkers (24), which showed a linear correlation between sexual activity and the androgenic hormonal profile during the menstrual cycle in women with and without a partner. In addition, the results of multiple regression analysis performed in our study have shown a strong relationship between changes in leptin and vaspin levels and changes in testosterone levels during the menstrual cycle. Further studies are necessary to explain this potential association. Our results, in line with those of previously published studies $(10,15,17,19)$, but contrary to others (16), revealed stable circulating adiponectin levels during the physiological menstrual cycle. Additionally, contrary to previously published results $(16,17,18,19)$, we observed that in the luteal phase, plasma adiponectin levels were inversely associated with circulating estradiol levels and proportional to $17-\mathrm{OH}$ progesterone concentrations. Thus, it seems that stable adiponectin levels during the physiological menstrual cycle are the effect of opposing actions of ovarian sex hormones. Furthermore, inverse associations between circulating adiponectin levels and waist circumference were shown, regardless of the menstrual cycle phase, which is consistent with the previous results (25). This observation suggests that visceral fat depot is a stronger factor affecting the amount of adiponectin in the circulation than ovarian hormones. The positive association between adiponectin and SHBG levels also indicates that this adipokine may play an indirect role in the regulation of bioavailability of estrogens and androgens in the midcycle and in the luteal phase. It should be noted that our study shows inverse correlations between serum SHBG and plasma adiponectin levels in all phases of the menstrual cycle. In addition, irrespective of the menstrual cycle phase, plasma leptin levels were proportional to body fat mass and fat percentage, while there were no associations between the remaining studied adipokines and anthropometric parameters. These results suggest that the visceral fat deposit is an important factor affecting adiponectin and SHBG levels in normal-weight women. Furthermore, it seems that changes of SHBG levels are directly associated with changes of adiponectin levels related to visceral fat deposit, while in general, fat deposit is more strongly associated with circulating leptin levels. It should be noted that BMI is a widely recognized parameter in the assessment of nutritional status; however, our data shows that using BMI as the only measure of fat stores can produce conflicting results. It is well known that visceral fat deposits are the place of steroid hormones' metabolism and the main source of adipokines and hormones $(26,27)$. It should also be mentioned that adipose tissue expresses androgen, estrogen and progesterone receptors (27).
On the other hand, probably due to low physical activity, there are increasing numbers of young women with normal BMI values who develop increased total and visceral fat deposits, known as metabolically obese normal weight (MONW) (28). Increased visceral fat deposit is a reason for excessive aromatase activity, inflammation, disturbances in the production and secretion of adipokines, as well as the development of insulin resistance $(29,30)$. Thus, all studies assessing the physiological aspect of the association between hormones of adipose tissue (adipokines) and sex hormones should include more accurate measures of nutritional status than BMI.

Additionally, our results, in accordance with a previous study (10) but contrary to another (15) revealed stable resistin levels during the menstrual cycle. Of interest, plasma resistin levels were associated with circulating LH and PRL levels in the midcycle. In addition, plasma resistin levels were proportional to the circulating PRL and inversely proportional to total testosterone concentrations in the luteal phase. It seems that despite the stable resistin levels during the menstrual cycle, this adipokine plays at least an indirect role in the regulation of reproductive function. This hypothesis is partially supported by our previous observation obtained in PCOS women, which revealed an inverse association between serum LH concentrations and plasma resistin levels (31). Our study provides a novel contribution by showing stable circulating levels of visfatin/NAMPT, omentin-1, apelin-36, RBP4 and IL-6 during the physiological menstrual cycle. Moreover, there were no associations between visfatin/NAMPT, omentin-1, apelin-36, RBP4 and IL- 6 and gonadotropins levels during the menstrual cycle. Similarly, these adipokines were not associated with either estradiol or $17-\mathrm{OH}$ progesterone concentrations in the follicular phase, with the exception of apelin-36, which was proportional to $17-\mathrm{OH}$ progesterone levels. While in the midcycle, circulating omentin-1 and apelin-36 were proportional to 17-OH progesterone level, in the luteal phase, visfatin/NAMPT and apelin-36 levels were inversely associated with estradiol concentrations. A previous study demonstrated the stimulating effect of estrogen on visfatin/NAMPT secretion by adipocytes (32). Interestingly, a different study was reported in which eutopic and ectopic endometrium apelin expression showed the cyclic changes (33); however, it should be noted that in our study, circulating apelin levels were stable during the menstrual cycle. Although there is a lack of data concerning the effect of estrogens on visfatin/NAMPT production in women, the visfatin/NAMPT correlation with androstendione suggests a potential role of this

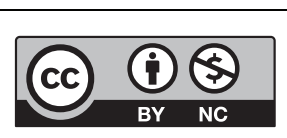

This work is licensed under a Creative Commons Attribution-NonCommercial 4.0 International License. 
adipokine in the regulation of adrenal gland function, which is difficult to explain. Furthermore, the exact role of visfatin/NAMPT on insulin resistance is doubtful.

One of the limitations of our study is the assessment of the parameters only three times in the cycle. A more robust study design would make assessments on every day of the menstrual cycle. Other limitations would be the lack of ultrasound monitoring of ovulation, and the indirect assessment of visceral fat deposits.

\section{Conclusions}

Our results revealed stable levels of adipokines during the physiological menstrual cycle, except for leptin and vaspin levels, which increased in both the midcycle and the luteal phases. The changes in leptin and vaspin levels were found to be significantly associated with changes in the secretion of testosterone, 17-OH progesterone and insulin, in the luteal phase.

\section{Declaration of interest}

The authors declare that there is no conflict of interest that could be perceived as prejudicing the impartiality of the research reported.

\section{Funding}

Research was funded by grant from the Medical University of Silesia.

\section{References}

1 Nishizawa H, Shimomura I, Kishida K, Maeda N, Kuriyama H, Nagaretani H, Matsuda M, Kondo H, Furuyama N, Kihara S, et al. Androgens decrease plasma adiponectin, and insulinsensitizing adipocyte-derived protein. Diabetes $2002512734-2741$. (doi:10.2337/diabetes.51.9.2734)

2 Chen BH, Song Y, Ding EL, Roberts CK, Manson JE, Rifai N, Buring JE, Gaziano JM \& Liu S. Circulating levels of resistin and risk of type 2 diabetes in men and women: results from two prospective cohorts. Diabetes Care 200932 329-334. (doi:10.2337/dc08-1625)

3 Körner A, Neef M, Friebe D, Erbs S, Kratzsch J, Dittrich K, Blüher S, Kapellen TM, Kovacs P, Stumvoll M, Blüher M, et al. Vaspin is related to gender, puberty and deteriorating insulin sensitivity in children. International Journal of Obesity 201135 578-586. (doi:10.1038/ ijo.2010.196)

4 De Souza Batista CM, Yang RZ, Lee MJ, Glynn NM, Yu DZ, Pray J, Ndubuizu K, Patil S, Schwartz A, Kligman M, et al. Omentin-1 plasma levels and gene expression are decreased in obesity. Diabetes 200756 1655-1661. (doi:10.2337/db06-1506)

5 Ulgen F, Herder C, Kühn MC, Willenberg HS, Schott M, Scherbaum WA \& Schinner S. Association of serum levels of retinolbinding protein 4 with male sex but not with insulin resistance in obese patients. Archives of Physiology and Biochemistry 2010116 57-62. (doi:10.3109/13813451003631421)

6 Psilopanagioti A, Papadaki H, Kranioti EF, Alexandrides TK \& Varakis JN. Expression of adiponectin and adiponectin receptors in human pituitary gland and brain. Neuroendocrinology 200989 38-47. (doi:10.1159/000151396)

7 Caprio M, Fabbrini E, Isidori AM, Aversa A \& Fabbri A. Leptin in reproduction. Trends in Endocrinology and Metabolism 200112 65-72. (doi:10.1016/S1043-2760(00)00352-0)

8 Reaux-Le Goazigo A, Alvear-Perez R, Zizzari P, Epelbaum J, BluetPajot MT \& Llorens-Cortes C. Cellular localization of apelin and its receptor in the anteriorpituitary: evidence for a direct stimulatory action of apelin on ACTH release. American Journal of Physiology: Endocrinology and Metabolism 2007292 E7-E15. (doi:10.1152/ ajpendo.00521.2005)

9 Riad-Gabriel MG, Jinagouda SD, Sharma A, Boyadjian R \& Saad MF Changes in plasma leptin during the menstrual cycle. European Journal of Endocrinology 199813 528-531. (doi:10.1530/eje.0.1390528)

10 Asimakopoulos B, Milousis A, Gioka T, Kabouromiti G, Gianisslis G, Troussa A, Simopoulou M, Katergari S, Tripsianis G \& Nikolettos N. Serum pattern of circulating adipokines throughout the physiological menstrual cycle. Endocrine Journal 200956 425-433. (doi:10.1507/ endocrj.K08E-222)

11 Wunder DM, Yared M, Bersinger NA, Widmer D, Kretschmer R \& Birkhäuser MH. Serum leptin and C-reactive protein levels in the physiological spontaneous menstrual cycle in reproductive age women. European Journal of Endocrinology 2006155 p137-p142. (doi:10.1530/eje.1.02178)

12 Capobianco G, de Muro P, Cherchi GM, Formato M, Lepedda AJ, Cigliano A, Zinellu E, Dessole F, Gordini L \& Dessole S. Plasma levels of C-reactive protein, leptin and glycosaminoglycans during spontaneous menstrual cycle: differences between ovulatory and anovulatory cycles. Archives of Gynecology and Obstetrics 2010282 207-213. (doi:10.1007/s00404-010-1432-2)

13 Al-Harithy RN, Al-Doghaither H \& Abualnaja K Correlation of leptin and sex hormones with endocrine changes in healthy Saudi women of different body weights. Annals of Saudi Medicine 200626 110-115. (doi:10.5144/0256-4947.2006.110)

14 Ludwig M, Klein HH, Diedrich K \& Ortmann O. Serum leptin concentrations throughout the menstrual cycle. Annals of Saudi Medicine 2000263 99-101.

15 Dafopoulos K, Sourlas D, Kallitsaris A, Pournaras S \& Messinis IE. Blood ghrelin, resistin, and adiponectin concentrations during the normal menstrual cycle. Fertility and Sterility 200992 1389-1394. (doi:10.1016/j.fertnstert.2008.07.1773)

16 Galván RE, Basurto L, Saucedo R, Campos S, Hernández M \& Zárate A. Adiponectin concentrations during menstrual cycle. Ginecologia Y Obstetricia De Mexico 20077 435-438.

17 Kleiblová P, Springer D \& Haluzík M. The influence of hormonal changes during menstrual cycle on serum adiponectin concentrations in healthy women. Physiological Research 200655 661-666.

18 Chalvatzas N, Dafopoulos K, Kosmas G, Kallitsaris A, Pournaras S \& Messinis IE. Effect of ovarian hormones on serum adiponectin and resistin concentrations. Fertility and Sterility 200991 1189-1194. (doi:10.1016/j.fertnstert.2008.01.027)

19 Hall N, White C \& O'Sullivan AJ. The relationship between adiponectin, progesterone, and temperature across the menstrual cycle. Journal of Endocrinological Investigation 200932 279-283. (doi:10.1007/BF03346467)

20 Rzymski P, Wilczak M, Malinger A, Włoszczak-Szubzda A, Jarosz MJ $\&$ Opala T. The interaction between menstrual cycle, Tumour Necrosis Factor alpha receptors and sex hormones in healthy non-obese women - results from an observational study. Annals of Agricultural and Environmental Medicine 201421 571-575. (doi:10.5604/12321966.1120604)

21 World Health Organisation. Obesity: preventing and managing the global epidemic. Report of a WHO Consultation (WHO Technical Report Series 894). Geneva, Switzerland: WHO, 2000. (available at: http://www.who.int/nutrition/publications/obesity/ WHO_TRS_894/en/)

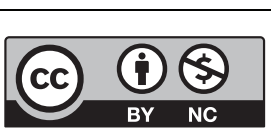

This work is licensed under a Creative Commons Attribution-NonCommercial 4.0 International License. 
22 Marsden PJ, Murdoch A \& Taylor R. Adipocyte insulin action during the normal menstrual cycle. Human Reproduction 199611 968-974. (doi:10.1093/oxfordjournals.humrep.a019333)

23 Escalante Pulido JM \& Alpizar Salazar M. Changes in insulin sensitivity, secretion and glucose effectiveness during menstrual cycle. Archives of Medical Research 199930 19-22. (doi:10.1016/S01880128(98)00008-6)

24 Caruso S, Agnello C, Malandrino C, Lo Presti L, Cicero C \& Cianci S. Do hormones influence women's sex? Sexual activity over the menstrual cycle. Journal of Sexual Medicine 201411 211-221. (doi:10.1111/jsm.12348)

25 Steffes MW, Gross MD, Schreiner PJ, Yu X, Hilner JE, Gingerich R \& Jacobs DR Jr. Serum adiponectin in young adults - interactions with central adiposity, circulating levels of glucose, and insulin resistance: the CARDIA study. Annals of Epidemiology 200414 492-498. (doi:10.1016/j.annepidem.2003.10.006)

26 Kershaw EE \& Flier JS Adipose tissue as an endocrine organ. Journal of Clinical Endocrinology and Metabolism 200489 2548-2556. (doi:10.1210/jc.2004-0395)

27 Siiteri PK. Adipose tissue as a source of hormones. American Journal of Clinical Nutrition 198745 277-282.
28 Bednarek-Tupikowska G, Stachowska B, Miazgowski T, KrzyżanowskaŚwiniarska B, Katra B, Jaworski M, Kuliczkowska-Płaksej J, JokielRokita A, Tupikowska M, Bolanowski M, et al. Evaluation of the prevalence of metabolic obesity and normal weight among the Polish population. Endokrynologia Polska 201263 447-455.

29 Killinger DW, Perel E, Daniilescu D, Kharlip L \& Lindsay WR. The relationship between aromatase activity and body fat distribution. Steroids 198750 61-72. (doi:10.1016/0039-128X(83)90062-4)

30 Olszanecka-Glinianowicz M \& Zahorska-Markiewicz B. Obesity as inflammatory disease. Postepy Higieny/Medycyny Doswiadczalnej 2008 62 249-257.

31 Olszanecka-Glinianowicz M, Kuglin D, Dąbkowska-Huć A \& Skałba P. Serum adiponectin and resistin in relation to insulin resistance and markers of hyperandrogenism in lean and obese women with polycystic ovary syndrome. European Journal of Obstetrics and Gynecology and Reproductive Biology 2011154 51-56. (doi:10.1016/j.ejogrb.2010.08.022)

32 Zhou J \& Seidel ER Estrogens induce visfatin expression in 3T3-L1 cells. Peptides 201031 271-274. (doi:10.1016/j.peptides.2009.12.004)

33 Ozkan ZS, Cilgin H, Simsek M, Cobanoglu B \& Ilhan N. Investigation of apelin expression in endometriosis. Journal of Reproduction and Infertility 201414 50-55.

Received in final form 25 September 2017

Accepted 6 October 2017 http://www.endocrineconnections.org DOI: 10.1530/EC-17-0186 (c) 2017 The authors Published by Bioscientifica Ltd

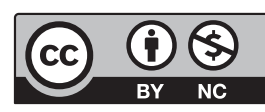

This work is licensed under a Creative Commons Attribution-NonCommercial 4.0 International License. 